\section{dCTP pyrophosphohydrase exhibits nucleic accumulation in multiple carcinomas}

\author{
Y. Zhang, ${ }^{1}$ W.Y. Ye, ${ }^{1}$ J.Q. Wang, ${ }^{1}$ \\ S.J. Wang, ${ }^{1}$ P. Ji, ${ }^{1}$ G.Y. Zhou, ${ }^{1}$ G.P. Zhao, ${ }^{2}$ \\ H.L. Ge, ${ }^{1}$ Y. Wang ${ }^{1,2}$ \\ 'Shanghai Institute of Immunology, \\ Department of Immunology, Institute \\ of Medical Sciences, Shanghai Jiao Tong \\ University School of Medicine \\ 2Shanghai Key Laboratory of Health and \\ Disease Genomics, Chinese National \\ Human Genome Center at \\ Shanghai/Shanghai Academy of Science \\ and Technology, China
}

\section{Abstract}

Nucleoside triphosphate pyrophosphohydrolase (NTP-PPase) functions as one of the mechanisms to guarantee the fidelity of DNA replication through the cleavage of non-canonical nucleotides into di- or monophosphates. Human NTP-PPase is poorly understood and investigated. In the present study, by using tissue microarrays with the paired cancer and adjacent regions, we found that with the prevalent expression of dCTP pyrophosphohydrase (DCTPP1) in the cytosol and nucleus in tumors investigated, DCTPP1 was inclined to accumulate in the nucleus of cancer cells compared to the paired adjacent tissue cells in multiple carcinomas including lung, breast, liver, cervical, gastric and esophagus cancer. More significantly, the higher DCTPP1 expression in the nucleus of lung, gastric and esophagus cancer cells was associated with histological subtypes. The nucleic accumulation of DCTPP1 was apparently observed as well when tumor cell line MCF-7 was treated with $\mathrm{H}_{2} \mathrm{O}_{2}$ in vitro. Considering the roles of DCTPP1 on restricting the concentration of non-canonical nucleotides in the nucleotide pool, accumulation of DCTPP1 in the nucleus of tumor cells might suffice for maintaining the proper DNA replication in order to fulfill the requirement for the survival and proliferation of tumor cells.

\section{Introduction}

Besides the essential role as precursors of DNA/RNA synthesis, free canonical nucleotides in living organisms play various roles to maintain physiological life activity, such as signal transmitters, molecular switches, coenzymes or energy carriers. Therefore, accumulation of non-canonical nucleotides, such as oxo- or methylated- deoxynucleoside triphosphates, in (d)NTPs pool is a prominent cause to reduce the fidelity of DNA replication, leading to the retardation of cell survival and proliferation. ${ }^{1}$ Non-canonical nucleotides are generated either as byproducts of cellular metabolism or by deamination or oxidation of bases in natural nucleotides. ${ }^{2}$ Incorporation of these non-canonical nucleotides during DNA replication results in the ambiguous base pairing and subsequent accumulation of mutations. The incorporated non-canonical nucleotides can be repaired by DNA repair machinery, ${ }^{3}$ such as mismatch repair (MMR) ${ }^{4}$ and base excision repair (BER). ${ }^{5}$ In addition, cells also develop elaborate enzymatic systems that maintain the purity of nucleotide pools. This is achieved by one class of enzymes named nucleoside triphosphate pyrophosphohydrolase (NTP-PPase) to eliminate the noncanonical nucleotides through cleaving them into di- or mono-phosphates. MutT-like proteins are the most extensively studied NTPPPase which are involved in degrading oxidized purine nucleotides, such as 8-0xo(d)GTP and 2-0H-dATP. ${ }^{2}$ A lack of mutT in Escherichia coli increases AT-CG mutation in both DNA and mRNA, ${ }^{6,7}$ while depletion of MTH1 in mice leads to a higher incidence of spontaneous tumorigenesis.

The MazG family enzymes belong to allalpha NTP-PPase including dimeric dUTPases, HisE and MazG, et al..$^{9}$ It is supposed that they might perform house-cleaning function by degrading abnormal (d)NTPs, such as dUTP, 2hydroxy-dATP and 8-0xo-dGTP, preventing them from incorporating into DNA or RNA.10 However, biological significance of MazG remains an enigma until recently. An essential role for MazG in larval molting and development is demonstrated in the parasitic roundworm Ascaris. ${ }^{11}$ In Caenorhabditis elegans, MazG is required for larval development and intestinal function. ${ }^{12}$ The study on MazG from Mycobacterium smegmatis demonstrates that it is required for full oxidative stress responses. ${ }^{13}$ The mouse RS21-C6 contains a typical conserved MazG domain. It can hydrolyze canonical dCTP into dCMP and prevent inappropriate DNA methylation, aberrant DNA replication or DNA mutagenesis by hydrolyzing modified dCTP. ${ }^{14}$

Human NTP-PPase is poorly understood and investigated. Inosine triphosphate pyrophosphatase is proved to be pivotal in maintaining genome stability and preventing apoptosis in human cells. ${ }^{9}$ A few studies have reported the associations of NTP-PPase with hyperthyroidism ${ }^{10}$ and neoplasms. ${ }^{10-13}$ dCTP pyrophosphohydrolase (DCTPP1) is a newly defined MazG ortholog in human. It is speculated to specifically hydrolyze 5-methyl-2-deoxy-cyti-
Correspondence: Ying Wang, Shanghai Institute of Immunology, Department of Immunology and Microbiology, Institute of Medical Sciences, School of Medicine, Shanghai Jiaotong University, 200025, China.

Tel./Fax: +86.21.63846383.

E-mail: ywang@sibs.ac.cn

Key words: dCTP pyrophosphohydrase, carcinomas, nucleic accumulation, immunohistochemistry, tissue microarrays.

Contributions: YZ, WYY, JQW performed the experiments; SJW, PJ performed the analysis; YZ, YW wrote the manuscript; GYZ, HLG supervised the experiment and manuscript; GPZ, YW designed the experiment and supervised the manuscript; all the authors read and approved the final manuscript version. YZ and WYY contributed equally to this work.

Acknowledgments: this work was supported by grants from Shanghai Municipal Health Bureau (20114195) and Shanghai Municipal Education Commission (13ZZ083).

Conflict of interests: the authors declare no competing interests.

Received for publication: 3 July 2013.

Accepted for publication: 2 September 2013.

This work is licensed under a Creative Commons Attribution NonCommercial 3.0 License (CC BYNC 3.0).

(C) Copyright Y. Zhang et al., 2013

Licensee PAGEPress, Italy

European Journal of Histochemistry 2013; 57:e29 doi:10.4081/ejh.2013.e29

dine triphosphate, probably avoiding the incorporation of non-canonical nucleotides into mitotic DNA and the silence of functional genes. Due to its potential roles in modulating nucleic acid metabolism, it might be engaged in multiple metabolic disorders including tumorigenesis which is worthwhile further investigating.

In this study, we have primarily investigated the DCTPP1 expression profiles in normal and cancerous tissues. We found that DCTPP1 was significantly accumulated in the nucleus of liver, breast, lung, gastric, esophagus cancer cells as well as cervical carcinoma, which implies the potential roles of DCTPP1 under malignant pathology.

\section{Materials and Methods}

\section{Tissue microarray}

All the tissue microarrays (TMA) under 
investigation were commercially purchased from Shanghai Biochip (Shanghai, China). On tissue microarrays, duplicates of 1-mm diameter cylinders from tumor regions and the paired adjacent regions with a total of four punches were included in each case to ensure reproducibility and homogeneous staining of the slides. Sections of $4-\mu \mathrm{m}$ thickness were placed on 3-amino-propyl-triethoxysilane-coated slides for subsequent staining. In our study, tissue microarrays with 89 cases of lung cancer (Cat\# OD-CT-RsLug03-002 and OD-CTRsLug01-007), 61 cases of breast cancer (Cat\# OD-CT-RpBre03-004 and OD-CT-RpBre03005), 31 cases of esophagus cancer (Cat\# ODCT-DgEso03-003), 31 cases of gastric cancer (Cat\# OD-CT-DgStm03-003), 31 cases of liver cancer (Cat\#OD-CT-DgLiv03-004), 31 cases of cervical cancer (Cat\# OD-CT-RpUtr03-003) and 29 cases of colorectal cancer (Cat\# OD-CTDgCol03-003) were subjected to the study. Matched pairs of cancer samples and adjacent tissues were included in all tissue microarrays.

The study was retrospective and was approved by the Institutional Review Board of Shanghai Jiaotong University School of Medicine.

\section{Immunohistochemistry}

The immunohistochemical protocols are described elsewhere. ${ }^{15}$ In brief, the tissue microarray slides were deparaffinized and hydrated routinely. Endogenous peroxidase was inhibited by incubating with freshly prepared PBS containing $3 \% \mathrm{H}_{2} \mathrm{O}_{2}$. The slides were treated in a microwave oven for $5 \mathrm{~min}$ in $0.01 \mathrm{M}$ citrate buffer ( $\mathrm{pH}$ 6.0) for antigen retrieval. After blocking with PBS containing $1 \%$ goat serum, rabbit anti-human DCTPP1 pAb (Cat\#AP2821a, Abgent, San Diego, CA, USA) at dilution of 1:200 were added for incubation at $4^{\circ} \mathrm{C}$ overnight. After the incubation with primary antibody, tissue microarray slides were sequentially incubated with biotinylated goat anti-rabbit IgG (Cat\# B8895; SigmaAldrich, St. Louis, MO, USA) and ExtrAvidinconjugated horseradish peroxidase (ExtrAvidin Peroxidase Staining Kit, Extra-2; Sigma-Aldrich) at dilutions of 1:800 and 1:30, respectively. Slides were developed with diaminobenzidine (DAB; Sigma-Aldrich) substrate and counterstained with hematoxylin, dehydrated, and mounted. For negative control in the IHC procedures, PBS with 10\% normal mouse serum was used for the substitution of primary antibody.

\section{Evaluation of immunohistochemical staining}

Slides were observed under the light microscope and scored by two experienced pathologists independently. For each sample, five fields were randomly selected. Total and nucleic staining of DCTPP1 was scored by conventional semi-quantitative scoring system. The extent of staining was scored according to the percentage of DCTPP1-positive cells as follows: + (1-24\%), ++ (25-49\%), +++ (50-74\%), or $++++(75-100 \%),{ }^{16}$ respectively. For data analysis, scored as + was considered lowexpression, other scores were considered high-expression.

\section{In vitro treatment of MCF-7 with $\mathrm{H}_{2} \mathrm{O}_{2}$}

Human breast cancer cell line MCF-7 was purchased for American Type Culture Collection (Manassas, VA, USA) and maintained routinely in RPMI-1640 complete medium with $10 \%$ fetal bovine serum (FBS) and 100 $\mu \mathrm{g} / \mathrm{mL}$ penicillin-streptomycin (Invitrogen, Carslbad, CA, USA). For in vitro treatment of $\mathrm{H}_{2} \mathrm{O}_{2}$, MCF-7 cells were seeded on the cover glasses in 24 -well cell culture plate $\left(5 \times 10^{4}\right.$ cells per well) in $500 \mu \mathrm{L}$ RPMI-1640 complete medium overnight. The cells were treated with $\mathrm{H}_{2} \mathrm{O}_{2}$ at concentration of $50 \mu \mathrm{M}$ in complete medium for 0,1 and $2 \mathrm{~h}$, respectively. After the treatment, cells were washed with PBS and fixed in pre-cold acetone for 5-10 min and subjected to immunohistochemical staining against DCTPP1 as described above.

\section{Western blotting}

MCF-7 cells with or without $\mathrm{H}_{2} \mathrm{O}_{2}$ treatment were collected by trypsinization and spin. Cells were lysed in lysis buffer [ $25 \mathrm{mM}$ Tris-HCl pH 7.6, $150 \mathrm{mM} \mathrm{NaCl}, 1 \% \mathrm{NP}-40,1 \%$ sodium deoxycholate, $0.1 \%$ SDS, and $1 \%$ protease inhibitor cocktail (Sigma-Aldrich)]. Protein concentrations were determined using the Bio-Rad protein assay kit (Bio-Rad, Hercules, CA, USA). The cell lysates were separated on $12 \%$ SDS-PAGE gels and transferred to a PVDF membrane (Millipore, Billerica, MA, USA). The membrane was incubated for $1 \mathrm{~h}$ at room temperature in TBST solution [10 mM Tris-HCl (pH 8.0), $150 \mathrm{mM} \mathrm{NaCl}$, and 0.05\% Tween-20] supplemented with $5 \%$ nonfat dry milk and probed overnight at $4^{\circ} \mathrm{C}$ with antibodies against human DCTPP1 pAb (Abgent Inc., San Diego, CA, USA) at dilutions of 1:500. Binding antibodies were visualized using a suitable secondary antibody conjugated to horseradish peroxidase (1:2000, Sigma-Aldrich) and enhanced chemiluminescence reagents (Thermo, Rockford, IL, USA). Antibody against tublin (Sigma-Aldrich) was used to normalize the quantity of the sample loading.

\section{Statistical analysis}

Analysis was performed with SPSS 16.0 software package (SPSS Inc., Chicago, IL, USA).
The intergroup differences were examined by using $\chi^{2}$-test or Fisher's exact test. A P value $<0.05$ was considered statistically significant.

\section{Results}

\section{DCTPP1 expression in multiple cancer tissue microarrays}

To explore the possible role of DCTPP1 in tumorigenesis, we first surveyed the DCTPP1 expression in the total regions of commercial tissue microarrays with paired cancer and the adjacent regions from multiple carcinomas. Tissue microarrays including 89 cases of lung cancer, 61 cases of breast cancer, 31 cases of gastric cancer, 31 cases of esophagus cancer, 31 cases of liver cancer, 31 cases of cervical cancer and 29 cases of colorectal cancer were under investigation in this study. For each case, a paired adjacent tissue was studied in parallel as control. Our results indicated that DCTPP1 displayed cytosol and nucleic expressing patterns in both cancer and adjacent regions. Through statistical analysis, we found that both in lung and breast cancer, DCTPP1 was significantly hyper-expressed in cancer cells compared to the adjacent regions. In other five types of cancer, the expression levels of DCTPP1 were comparable between paired cancer and adjacent regions (Table 1).

\section{Higher nucleic DCTPP1 expressing levels in cancer cells from multiple carcinomas}

During our study, a very interesting phenomenon was observed that DCTPP1 in most of the cancer cells was highly expressed in the nucleus when examined precisely in tissue microarrays. However, in the adjacent regions the nucleic expression level of DCTPP1 was less apparent. Considering the fundamental role of pyrophosphotase in controlling the elimination of non-canonical nucleotides, it is rationale that the expression of DCTPP1 in the nucleus is corresponding to its biological function. Whether the nucleic DCTPP1 expression is different between the cancer and the adjacent tissue cells was further studied in tissue microarrays. Our results indicated that the frequency of positive DCTPP1 staining in the nucleus of six types of cancer cells was higher than that in tissues adjacent to tumors (Table 2 ). For instance, in lung cancer tissue microarray three out of 89 tissues adjacent to the cancer cells showed high nucleic DCTPP1 staining. Among the 89 paired lung cancer tissues, 68 cases exhibited strong positive staining in the nucleus and the rest 21 were weak for DCTPP1 (Figure 1). As shown in Table 2, there 
were significant differences in terms of the number of cases with high nucleic DCTPP1 expression between the lung cancer and the adjacent group $(76.4 \%$ vs $3.3 \%, \mathrm{P}=0.000)$. In breast cancer, the high expression rate of nucleic DCTPP1 was $93.4 \%$ (57/61) in the cancer cells while it was only $54.1 \%(33 / 61)$ in the adjacent normal cells (Table 2; Figure 2), indicating dramatically difference of high nucleic DCTPP1 expression between the breast cancer and the adjacent controls $(\mathrm{P}=0.000)$. In liver carcinoma, 15 out of 31 tumor tissues were strong positive DCTPP1 staining in the nucleus whereas 1 out of 31 adjacent tissues displayed strong expression (Figure 2). The number of cases with high nucleic DCTPP1 expression between the liver cancer and the adjacent controls ( $48.4 \%$ vs $3.2 \%, \mathrm{P}=0.000$ ) was also dramatically different. Cervical cancer was another type of carcinoma that displayed significant difference of high nucleic DCTPP1 expression between cancer cells and the adja- cent tissue cells $(67.7 \%$ vs $25.8 \%, \mathrm{P}<0.001)$ (Figure 2). The difference is not so dramatic but still significant between the gastric carcinoma and the paired adjacent controls $(87.1 \%$ vs $61.3 \%, \mathrm{P}=0.02$ ) (Figure 3 ) as well as in esophagus carcinoma $(67.7 \%$ vs $41.9 \%$, $\mathrm{P}=0.048$ ) (Figure 4). However, there was no significant difference of high nucleic DCTPP1 expression in colorectal cancer cells when compared to the paired adjacent cells $(\mathrm{P}>0.05)$ (Table 2). These results indicated that nucleic

Table 1. DCTPP1 expression in seven types of cancer and the paired adjacent regions in tissue microarrays.

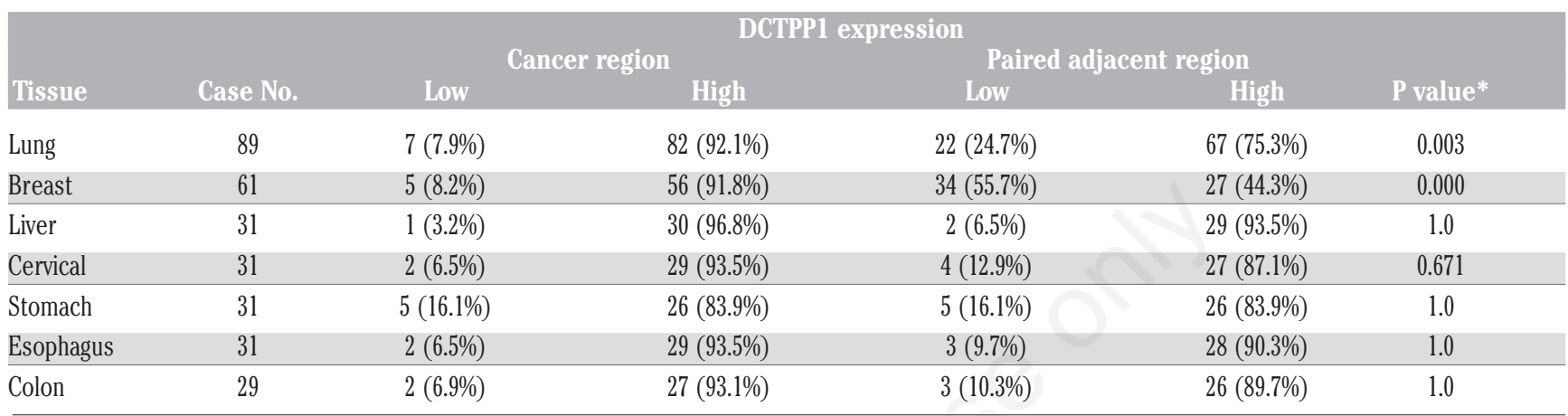

*High expression of DCTPP1 in cancer region vs the paired adjacent region. The high expression of DCTPP1 is defined as the percentage of DCTPP1-positive cells more than 25\% observed.

Table 2. Nucleic DCTPP1 expression in seven types of cancer tissues and the paired adjacent regions.

\begin{tabular}{|c|c|c|c|c|c|c|}
\hline \multirow[b]{3}{*}{ Tissue } & \multirow[b]{3}{*}{ Case No. } & \multicolumn{4}{|c|}{ nucleic DCTPP1 expression } & \multirow[b]{3}{*}{ P value* } \\
\hline & & \multicolumn{2}{|c|}{ Cancer region } & \multicolumn{2}{|c|}{ Paired adjacent region } & \\
\hline & & Low & High & Low & High & \\
\hline Lung & 89 & $21(23.6 \%)$ & $68(76.4 \%)$ & $86(96.6 \%)$ & $3(3.4 \%)$ & 0.000 \\
\hline Breast & 61 & $4(6.6 \%)$ & $57(93.4 \%)$ & $28(45.9 \%)$ & $33(54.1 \%)$ & 0.000 \\
\hline Liver & 31 & $16(51.6 \%)$ & $15(48.4 \%)$ & $30(96.8 \%)$ & $1(3.2 \%)$ & 0.000 \\
\hline Cervical & 31 & $10(32.3 \%)$ & $21(67.7 \%)$ & $23(74.2 \%)$ & $8(25.8 \%)$ & 0.001 \\
\hline Stomach & 31 & $4(12.9 \%)$ & $27(87.1 \%)$ & $12(38.7 \%)$ & $19(61.3 \%)$ & 0.02 \\
\hline Esophagus & 31 & $10(32.3 \%)$ & $21(67.7 \%)$ & $18(58.1 \%)$ & $13(41.9 \%)$ & 0.048 \\
\hline Colon & 29 & $4(13.8 \%)$ & $25(86.2 \%)$ & $10(34.5 \%)$ & $19(65.5 \%)$ & 0.053 \\
\hline
\end{tabular}

*High expression of DCTPP1 in cancer region us the paired adjacent region. The high expression of DCTPP1 is defined as the percentage of DCTPP1-positive cells more than 25\% observed.

Table 3. Association of clinicopathological features of lung cancer, gastric cancer and esophagus cancer with nucleic DCTPP1 expression.

\begin{tabular}{|c|c|c|c|c|c|c|c|c|c|c|c|c|}
\hline \multirow[b]{2}{*}{$\begin{array}{l}\text { Clinicopathologic } \\
\text { parameters }\end{array}$} & \multicolumn{3}{|c|}{$\begin{array}{c}\text { Lung cancer } \\
\text { Nucleic DCTPP1 expression (\%) }\end{array}$} & \multicolumn{4}{|c|}{$\begin{array}{c}\text { Gastric cancer } \\
\text { Nucleic DCTPP1 expression (\%) }\end{array}$} & \multirow[b]{2}{*}{$\mathbf{P}$} & \multicolumn{4}{|c|}{$\begin{array}{c}\text { Esophagus cancer } \\
\text { Nucleic DCTPP1 expression (\%) }\end{array}$} \\
\hline & Case No. & Low & High & $\mathbf{P}$ & Case No. & Low & High & & Case No. & Low & High & $\mathbf{P}$ \\
\hline \multicolumn{13}{|l|}{ Age } \\
\hline$<60$ & 32 & $7(21.9 \%)$ & $25(78.1 \%)$ & 0.768 & 15 & $2(13.3 \%)$ & $13(86.7 \%)$ & 0.613 & 18 & $7(38.9 \%)$ & $11(61.1 \%)$ & 0.694 \\
\hline$\geq 60$ & 57 & $14(24.6 \%)$ & $43(75.4 \%)$ & & 16 & $3(18.8 \%)$ & $13(81.2 \%)$ & & 13 & $3(23.1 \%)$ & $10(76.9 \%)$ & \\
\hline \multicolumn{13}{|l|}{ Gender } \\
\hline Male & 69 & $16(23.2 \%)$ & $53(76.8 \%)$ & 0.775 & 21 & $2(9.5 \%)$ & $19(90.5 \%)$ & 0.577 & 27 & $9(33.3 \%)$ & $18(66.7 \%)$ & 1.000 \\
\hline Female & 20 & $5(25.0 \%)$ & $15(75.0 \%)$ & & 10 & $2(20.0 \%)$ & $8(80.0 \%)$ & & 4 & $1(25.0 \%)$ & $\%)$ & \\
\hline \multicolumn{13}{|l|}{ Histological subtype } \\
\hline SCLC & 8 & $0(0)$ & $8(100 \%)$ & $0.190 * \mathrm{SC}$ & 6 & $3(50.0 \%)$ & $3(50.0 \%)$ & 0.023 & & $4(44.4 \%)$ & $5(55.6 \%)$ & 0.032 \\
\hline LCC (NSCLC) & 10 & $2(20.0 \%)$ & $8(80.0 \%)$ & $0.016^{* *} \mathrm{AC}$ & 12 & $1(8.3 \%)$ & $11(91.7 \%)$ & & AC 15 & $2(13.3 \%)$ & $13(86.7 \%)$ & \\
\hline SC (NSCLC) & 41 & $15(36.6 \%)$ & $26(63.4 \%)$ & $\mathrm{UC}$ & 6 & $0(0)$ & $6(100 \%)$ & & SCC 6 & $4(66.7 \%)$ & $2(33.3 \%)$ & \\
\hline SAC (NSCLC) & 10 & $1(10.0 \%)$ & $9(90.0 \%)$ & SR & 7 & $0(0)$ & $7(100 \%)$ & & UC 1 & $0(0)$ & $1(100 \%)$ & \\
\hline AC (NSCLC) & 20 & $3(15.0 \%)$ & $17(85.0 \%)$ & & & & & & & & & \\
\hline
\end{tabular}

SCLC, small cell lung cancer; NSCLC, non small cell lung cancer; LCC, large cell carcinoma; SC, squamous carcinoma; SAC, squamous adeno carcinoma; $\mathrm{AC}$, = adenocarcinoma; SCC, small cell cancer; UC, undifferentiated carcinoma; SR, signet ring cell carcinoma. ${ }^{*}$ Compared between SCLC and NSCLC; ${ }^{* *}$ compared among subtypes of NSCLC. 
expression of DCTPP1 was more remarkable in cancer cells although the overall expression level of DCTPP1 was similar in cancer and the paired adjacent tissue regions.

\section{Association of nucleic DCTPP1 expression with clinic pathologic features}

To further evaluate the significance of DCTPP1 in tumor, the clinical, epidemiologic, and histopathologic characteristics of patients with lung cancer, gastric cancer and esophagus cancer, and the association of high nucleic expression of DCTPP1 with the clinicopathologic parameters were analyzed. As shown in Table 3 , there were no statistically significant associations in the high nucleic expression of DCTPP1 with regard to patients' age and gender. However, the correlation between histological subtypes and the levels of DCTPP1 in non-small cell lung cancer $(\mathrm{P}=0.016)$, gastric cancer $(\mathrm{P}=0.023)$ or esophagus cancer $(\mathrm{P}=0.032)$ were statistically striking. For instance, in gastric cancer patients, the nucleic DCTPP1 expression was significantly correlated with histological subtypes. DCTPP1 expression was higher in signet ring cell carcinoma $(7 / 7)$ and undifferentiated carcinoma (6/6) than in tubular adenocarcinoma (11/12) or squamous carcinoma (3/6). In esophagus cancer tissue samples, the percentages of high nucleic DCTPP1 expression in adenocarcinoma, squamous carcinoma and small cell cancer patients were $91.7 \%$ (11/12), 55.6\% (5/9) and $33.3 \%$ (2/6), respectively. In lung cancer, the nucleic DCTPP1 was highly expressed in all small cell lung cancer (SCLC) patients (10/10) whereas only 60 out of 81 non-SCLC patients displayed high DCTPP1 nucleic expression.

When we subgrouped the non-SCLC patients into squamous carcinoma, adenocarcinoma, squamous adenocarcinoma and large cell carcinoma subtypes, the expressing rate of high DCTPP1 expression in the nucleus was lowest in squamous carcinoma patients (26/41) whereas comparable among other three groups. In addition, no strong correlations in the nucleic accumulation of DCTPP1 with clinicopathology were detectable in breast cancer, liver cancer, colon cancer and cervical cancer patients (data not shown).

\section{Nucleic accumulation of DCTPP1 upon $\mathrm{H}_{2} \mathrm{O}_{2}$ treatment in vitro}

To further investigate the feasibility of DCTPP1 nucleic accumulation, a breast cancer cell line MCF-7 was subjected to be stimulated with $\mathrm{H}_{2} \mathrm{O}_{2}$ for different duration. Expression of DCTPP1 was determined by immunohistochemical staining and western blotting. As shown in Figure 5A, DCTPP1 expression was up-regulated in MCF-7 upon $\mathrm{H}_{2} \mathrm{O}_{2}$ stimulation after 1-hour-treatment. When looking closely at the distribution of DCTPP1 in MCF-7 cells, we observed that DCTPP1 molecules started to accumulate in the nucleus after one hour's treatment (Figure $5 \mathrm{~B}-\mathrm{c}$ ) and the tendency was more apparent at $2 \mathrm{~h}$ (Figure $5 \mathrm{~B}-\mathrm{d}$ ), which demonstrated the occurrence of nucleic accumulation of DCTPP1 when encountering extra stress signals.

\section{Discussion}

In the present study we have, for the first time, explored the expression pattern of
DCTPP1 in multiple carcinomas. Our results revealed the hyper-expression property in the nucleus, suggesting nucleus accumulation of DCTPP1 in cancerous tissues compared to the corresponding adjacent tissues investigated.

NTP-PPase is supposed to play an important role in regulating DNA replication and energy metabolism. Members of the NTP-PPase family have been shown to eliminate non-canonical nucleotides from the intracellular NTP pools. For example, dUTPase hydrolyzes deoxyuridine triphosphate while MTH1 hydrolyzes oxidized purine nucleotide triphosphates, including 8-oxo-(deoxy) guanosine triphosphate (8oxo-(d)GTP) and 2-hydroxy-(deoxy) adenosine triphosphate (2-0H-(d)ATP). ${ }^{17}$ In cancer cells, the elevated NTP-PPase activity will probably

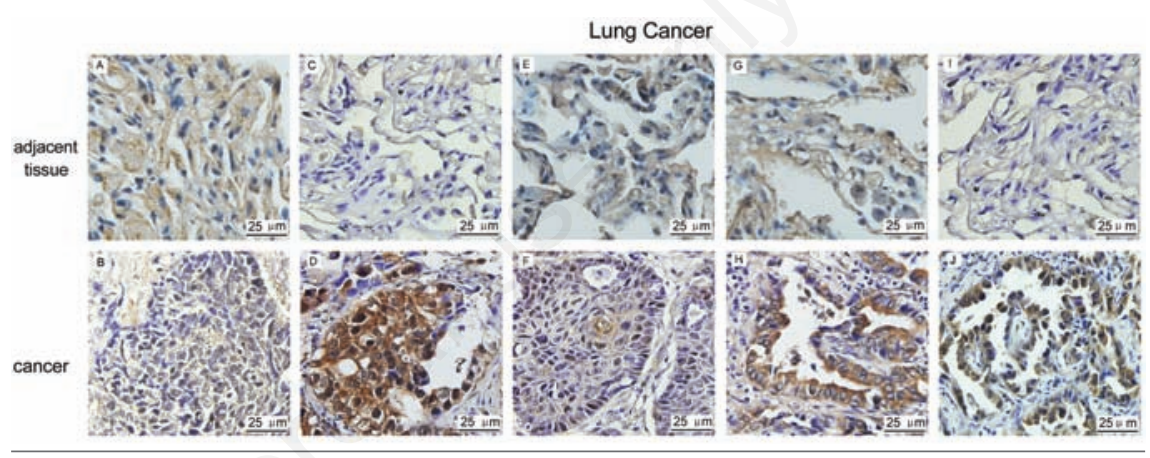

Figure 1. Representative examples of immunohistochemical staining of DCTPP1 in lung cancer with different histological subtypes. DCTPP1 staining was determined simultaneously in tissue arrays with caner and paired adjacent tissue regions. Positive staining of DCTPP1 is shown in dark brown while the nucleus is stained with H\&E dye. A) paired adjacent region of SCLC; B) SCLC; C) paired adjacent region of LCC; D) LCC; E) paired adjacent region of squamous carcinoma; F) squamous carcinoma; G) paired adjacent region of SAC; H) SAC; I) paired adjacent region of adenocarcinoma; J) adenocarcinoma. Magnification 400×; scale bar: $25 \mu \mathrm{m}$. SCLC, small cell lung cancer; LCC, large cell carcinoma; SAC, squamous adenocarcinoma.

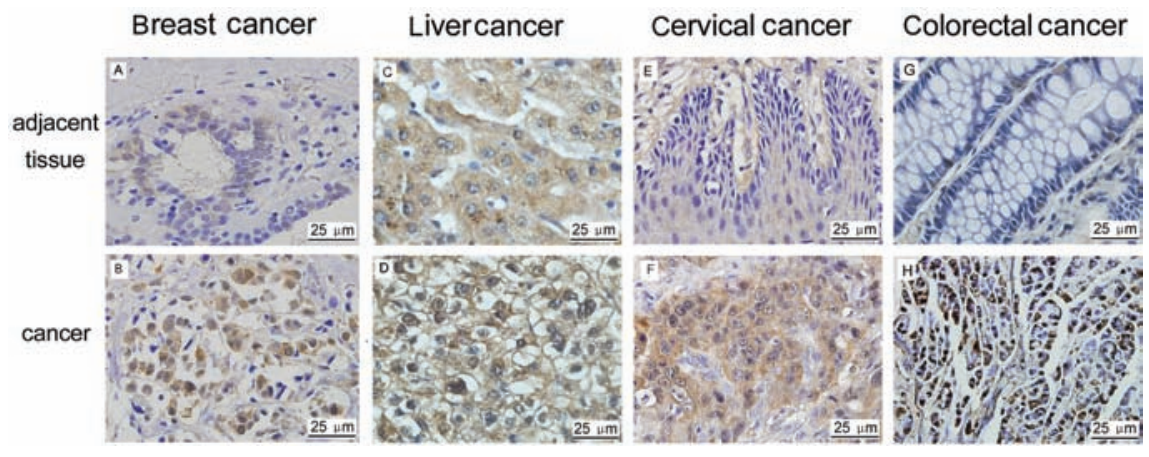

Figure 2. Representative examples of immunohistochemical staining of DCTPP1 in breast cancer, liver cancer, cervical cancer and colorectal cancer. DCTPP1 staining was determined simultaneously in tissue arrays with caner and paired adjacent tissue regions. Positive staining of DCTPP1 is shown in dark brown while nucleus is stained with $\mathrm{H} \& \mathrm{E}$ dye. DCTPP1 was slightly expressed in: A) paired adjacent tissues of breast cancer; C), liver cancer; E) cervical cancer; G) colorectal cancer; DCTPP1 was highly expressed in: B) breast infiltrative ductal carcinoma; D), hepatocellular carcinoma tissues; F), squamous cell cervical carcinoma; H) mucinous colon adenocarcinoma. Magnification: $400 \times$; scale bar: $25 \mu \mathrm{m}$. 
lower the concentration of non-canonical nucleotides, prevent their aberrant incorporations into DNA and thus might make the cancer cells more resistance to DNA damage and apoptosis. Indeed, the overexpression of certain pyrophosphatases has recently been reported in gastric cancer, ${ }^{18}$ prostate cancer, ${ }^{19}$ hepatocellular carcinoma ${ }^{20}$ and brain cancer. ${ }^{21}$ In the present study, we have surveyed the prevalent expression of DCTPP1, a dCTP pyrophosphohydrase in multiple carcinomas. Consistent with above mentioned previous studies, we have also observed the hyperexpression in lung and breast cancer, which provides another evidence to support the potential involvement of NTP-PPase in tumor.

Nucleic accumulation of DCTPP1 in cancer cells described here is another striking phenomenon. Nucleic translocation of molecules such as transcription factors from cytosol or membrane is mostly associated with cell proliferation and differentiation upon stress, immune stimuli as well as danger signals..$^{22,23}$ In tumorigenesis, many tumor-associated molecules including $\mathrm{p} 53,{ }^{24}$ HER-2, ${ }^{25}$ androgen receptor, ${ }^{26}$ nuclear transcription factors like $\mathrm{NF}-\mathrm{KB}^{27}$ etc., are reported to perform the aberrant nucleic translocation which are demonstrated to be critical for the proliferation, mitosis and transformation of tumor cells. In the present study, nucleic accumulation of DCTPP1 in cancer cells investigated clearly meets its putative functionality in guaranteeing the correct DNA synthesis under mitosis of tumor cells. To our knowledge, this is the first report describing the association between NTP-PPase nucleus distribution and tumor. Our further study indicates that under oxidative stress DCTPP1 accumulates in the nucleus which is consistent with its house-cleaning function. Although it is not exactly the same situation compared to tumorigenesis or progression, we still speculate that DCTPP1 overexpression in the nucleus meets the requirement for tumorigenesis through adjusting the nucleotide metabolism with less non-canonical nucleotide concentration in the nucleotide pool. It needs further investigation to understand why and how DCTPP1 accumulates into the nucleus under pathological circumstances.

In this study, there was no significant difference of high nucleic DCTPP1 expression in colorectal cancer cells when compared to the paired adjacent tissue cells. One of the possibilities is due to the small numbers of colorectal tissue samples in tissue microarrays we have investigated $(n=29)$. In addition, it is necessary to classify the samples according to histopathology. In fact, we have found that the nucleic DCTPP1 was significantly overexpressed in the adenocarcinoma than in squamous carcinoma $(\mathrm{P}<0.05)$ in our study (data not shown). According to our study,
DCTPP1 expression is diverse in different histological subtypes of lung, gastric and esophagus cancer. Although it is hard to bridge the malignancy and the histological subtypes in clinic, one of the key orientations of histological subtyping lies in the determination of chemotherapy strategy for the patients. For instance, in lung cancer, small cell lung cancer (SCLC) is more sensitive to chemotherapy than non-small cell lung cancer (NSCLC). Among NSCLC, patients with squamous, adenocarcinoma or squamous adenocarcinoma lung cancer also undergo diverse clinical chemotherapy combination. Our discovery on the association of DCTPP1 with histological subtypes thus raises another possibility that with the different expression levels of DCTPP1 in tumors of different histological subtypes,
DCTPP1 might be a potential new indicator to evaluate the efficacy of chemo-sensitivity against tumor. If so, to develop chemical compounds targeting DCTPP1 activity will probably improve the anti-tumor chemotherapy. In fact, it has already been reported that the inhibition of human deoxyuridine triphosphatase (dUTPase) has become a promising approach to enhance the efficacy of 5-fluorouracil (5FU)-based chemotherapy in vivo. ${ }^{28}$ In the future, molecular dissection of DCTPP1 in tumorigenesis and progression will accelerate the potential application of DCTPP1 as a novel target for cancer therapy.

What the biological role of DCTPP1 is in mammalian cells remains poorly understood. In normal cells, DCTPP1 is speculated to catalyse the hydrolysis of dCTP to dCMP in order to

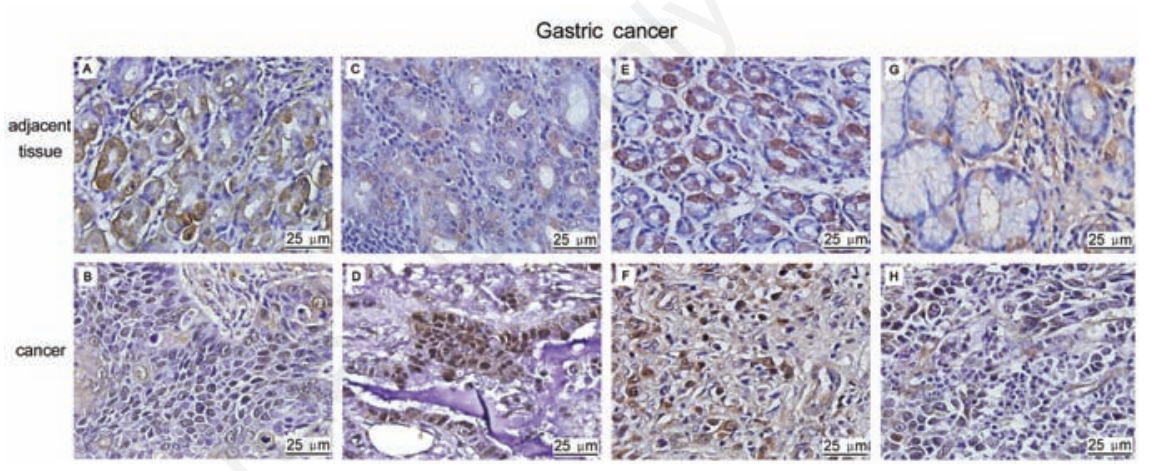

Figure 3. Representative examples of immunohistochemical staining of DCTPP1 in gastric cancer with different histological subtypes. DCTPP1 staining was determined simultaneously in tissue arrays with cancer and the paired adjacent tissue regions. Positive staining of DCTPP1 is shown in dark brown while the nucleus is stained with $H \& E$ dye. In chronic superficial gastritis tissue (A, C, E and G), the expression of DCTPP1 was slight both in the cytosol and nucleus. In the paired cancer tissues, such as: B) squamous cell carcinoma; D) adenocarcinoma; F) signet ring cell carcinoma; H) undifferentiated carcinoma, DCTPP1 was found highly expressed in the nucleus of cancer cells. Magnification: 400x; scale bar: $25 \mu \mathrm{m}$.

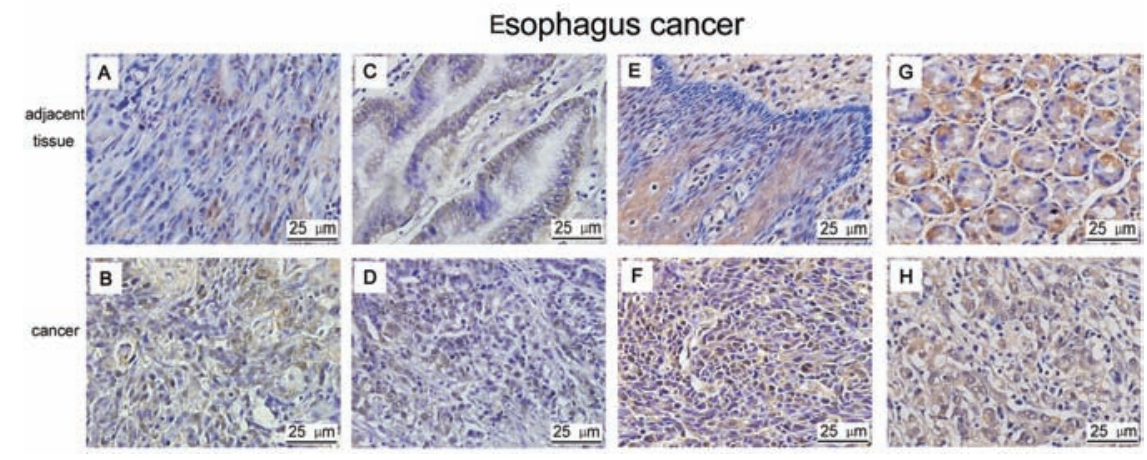

Figure 4. Representative examples of immunohistochemical staining of DCTPP1 in esophagus cancer with different histological subtypes. DCTPP1 staining was determined simultaneously in tissue microarrays with caner and the paired adjacent tissue regions. Positive staining of DCTPP1 is shown in dark brown while nucleus is stained with H\&E dye. In the paired adjacent tissues (A, C, E and G), the expression of DCTPP1 was apparent both in the cytosol and the nucleus. In squamous cell carcinoma(B), adenocarcinoma (D), small cell cancer (F) and undifferentiated carcinoma (H), DCTPP1 was found highly expressed in the nucleus of cancer cells. Magnification: 400x; scale bar: $25 \mu \mathrm{m}$. 
maintain the dCMP pool at a certain level for thymidylate synthesis. ${ }^{14} \mathrm{dCMP}$ is the main precursor of dTMP, which is converted to dTTP subsequently by a two-step phosphorylation reaction..$^{29,30}$ It is reported that an imbalanced $\mathrm{dCTP} / \mathrm{dTTP}$ ratio in hamster fibroblasts mildly affect the fidelity of DNA replication. ${ }^{31}$ To maintain the proper ratio of dCTP/dTTP in the nucleotide pool seems important for physiological homeostasis. The activity of DCTPP1 in cells might, to some extent, be engaged in the modulation of the dCTP concentration in the nucleotide pool, which finally affects the ratio of $\mathrm{dCTP} / \mathrm{dTTP}$ in the intracellular nucleotide pool.

More interesting is the biological function of DCTPP1 to prevent non-canonical 5-methyldCTP from the incorporation into newly synthesized DNA. This might be linked to the regulation of gene expression by dCTP methylation level. It has been reported that inflammation-mediated halogenated cytosine damage products can mimic 5-methyl-cytosine in directing enzymatic DNA methylation and enhancing the binding to methyl-binding proteins. ${ }^{32}$ The overwhelming 5 -halogenated dCTP generated in chronic inflammatory tissues might be incorporated into promoter regions of important genes, like tumor-suppressor genes, inducing their silencing through inappropriate $\mathrm{CpG}$ methylation of the promoter regions or by binding to methyl-CpG-binding protein 2 and resulting in the transformation of normal tissue cells. In our study, we have found that nucleic DCTPP1 was also overexpressed in the inflammatory sites in gastric cancer and colorectal cancer (Figures 2G and 3A,E). We therefore propose that the nucleic expression of DCTPP1 to hydrolyze the modified deoxynucleotides might be one of the mechanisms to protect normal cells from the malignant transformation through degradation of cytosine damage products under inflammation.

In conclusion, the findings presented here indicate that aberrant nucleic DCTPP1 accumulation represents the common feature in

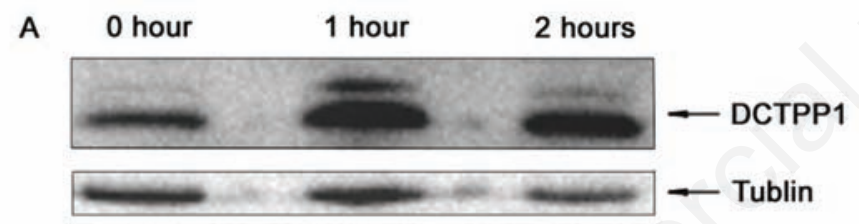

B
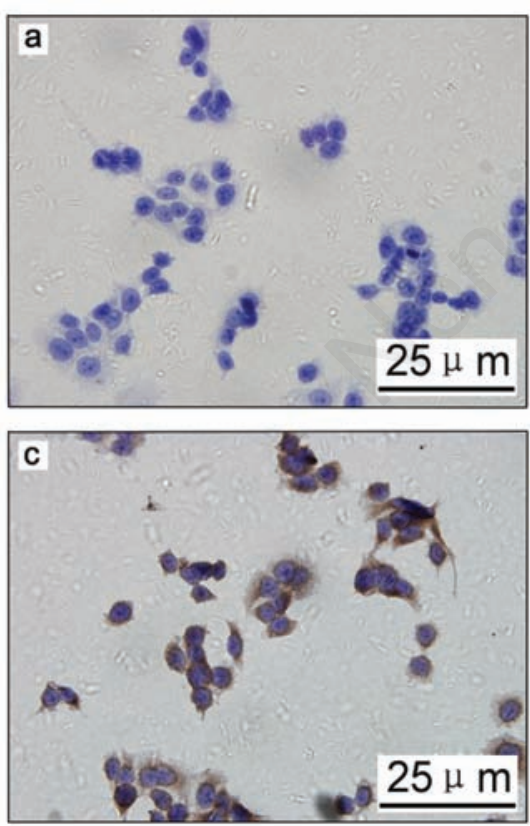

Figure 5. DCTPP1 expression in MCF-7 upon $\mathrm{H}_{2} \mathrm{O}_{2}$ treatment. MCF-7 was treated with $\mathrm{H}_{2} \mathrm{O}_{2}$ as described in the Materials and Methods section. DCTPP1 expression was determined by: A) western blotting; B) immunohistochemical staining. In Panel B, the slide was labeled with negative control (a) or anti-DCTPP1 antibody without $\mathrm{H}_{2} \mathrm{O}_{2}$ treatment (b), with 1-hour-treatment (c) and 2-hour-treatment (d), respectively. In immunohistochemcial assay, DCTPP1 was shown in dark brown while the nucleus was counterstained with H\&E dye. Magnification: 400×; scale bar: $25 \mu \mathrm{m}$. cancers, suggesting the role of DCTPP1 in regulating the nucleotide metabolism while displaying its potential application in diagnosis and therapy in the future.

\section{References}

1. Nakabeppu Y, Oka S, Sheng Z, Tsuchimoto D, Sakumi K. Programmed cell death triggered by nucleotide pool damage and its prevention by MutT homolog-1 (MTH1) with oxidized purine nucleoside triphosphatase. Mutat Res 2010;703:51-8.

2. Bessman MJ, Frick DN, O'Handley SF. The MutT proteins or "nudix" hydrolases, a family of versatile, widely distributed "housecleaning" enzymes. J Biol Chem 1996;271:25059-62.

3. Negrini S, Gorgoulis VG, Halazonetis TD. Genomic instability - an evolving hallmark of cancer. Nat Rev Mol Cell Biol 2010; 11:220-8.

4. Jiricny J. The multifaceted mismatchrepair system. Nat Rev Mol Cell Biol 2006; 7:335-46.

5. Lindahl T, Barnes DE. Repair of endogenous DNA damage. Cold Spring Harb Symp Quant Biol 2000;65:127-33.

6. Maki H, Sekiguchi M. MutT protein specifically hydrolyses a potent mutagenic substrate for DNA synthesis. Nature 1992; 355:273-5.

7. Taddei F, Hayakawa H, Bouton MF, Cirinesi AM, Matic I, Sekiguchi M, et al. Counteraction by MutT protein of transcriptional errors caused by oxidative damage. Science 1997;278:128-30.

8. Tsuzuki T, Egashira A, Igarashi $\mathrm{H}$, Iwakuma T, Nakatsura Y, Tominaga Y, et al. Spontaneous tumorigenesis in mice defective in the MTH1 gene encoding 8oxo-dGTPase. Proc Natl Acad Sci USA 2001;98:11456-61.

9. Moroz OV, Murzin AG, Makarova KS, Koonin EV, Wilson KS, Galperin MY. Dimeric dUTPases, HisE, and MazG belong to a new superfamily of all-alpha NTP pyrophosphohydrolases with potential "house-cleaning" functions. J Mol Biol 2005; 347:243-55.

10. Galperin MY, Moroz OV, Wilson KS, Murzin AG. House cleaning, a part of good housekeeping. Mol Microbiol 2006;59: 5-19.

11. Islam MK, Miyoshi T, Yamada M, Tsuji N. Pyrophosphatase of the roundworm Ascaris suum plays an essential role in the worm's molting and development. Infect Immun 2005;73:1995-2004.

12. Ko KM, Lee W, Yu J-R, Ahnn J. PYP-1, inorganic pyrophosphatase, is required for larval development and intestinal function in 
C-elegans. FEBS Lett 2007;581:5445-53.

13. Lu L-d, Sun Q, Fan X-y, Zhong Y, Yao Y-f, Zhao G-P. Mycobacterial MazG Is a Novel NTP Pyrophosphohydrolase Involved in Oxidative Stress Response. J Biol Chem 2010;285:28076-85.

14. Nonaka M, Tsuchimoto D, Sakumi K, Nakabeppu Y. Mouse RS21-C6 is a mammalian 2 '-deoxycytidine 5 '-triphosphate pyrophosphohydrolase that prefers 5iodocytosine. FEBS J 2009;276:1654-66.

15. Qian Y-B, Zhang J-B, Wu W-Z, Fang H-B, Jia W-D, Zhuang P-Y, et al. P48 is a predictive marker for outcome of postoperative interferon-alpha treatment in patients with hepatitis B virus infection-related hepatocellular carcinoma. Cancer 2006;107:1562-9.

16. Kimura M, Tsuda H, Morita D, Ichikura T, Ogata S, Aida S, et al. A proposal for diagnostically meaningful criteria to classify increased epidermal growth factor receptor and c-erbB-2 gene copy numbers in gastric carcinoma, based on correlation of fluorescence in situ hybridization and immunohistochemical measurements. Virchows Arch 2004;445:255-62.

17. Nakabeppu Y. Molecular genetics and structural biology of human MutT homolog, MTH1. Mutat Res 2001;477:5970 .

18. Jeong S-H, Ko GH, Cho YH, Lee Y-J, Cho BI, Ha W-S, et al. Pyrophosphatase overexpression is associated with cell migration, invasion, and poor prognosis in gastric cancer. Tumor Biology 2012;33:1889-98.

19. Lexander H, Palmberg C, Auer G,
Hellstrom M, Franzen B, Jornvall H, et al. Proteomic analysis of protein expression in prostate cancer. Anal Quant Cytol Histol 2005;27:263-72.

20. Takatori H, Yamashita T, Honda M, Nishino R, Arai K, Yamashita T, et al. dUTP pyrophosphatase expression correlates with a poor prognosis in hepatocellular carcinoma. Liver Int 2010;30:438-46.

21. Romeike BFM, Bockeler A, Kremmer E, Sommer P, Grasser F, Krick C. Immunohistochemical detection of dUTPase in intracranial tumors. Pathol Res Pract 2005;201:727-32.

22. Yang W, Zheng Y, Xia Y, Ji H, Chen X, Guo F, et al. ERK1/2-dependent phosphorylation and nuclear translocation of PKM2 promotes the Warburg effect. Nat Cell Biol 2012;14:1295-304.

23. Lin Y-Y, Hsu Y-H, Huang H-Y, Shann Y-J, Huang C-YF, Wei S-C, et al. Aberrant nuclear localization of EBP50 promotes colorectal carcinogenesis in xenotransplanted mice by modulating TCF-1 and beta-catenin interactions. J Clin Invest 2012;122:1881-94.

24. Muller PAJ, Vousden KH. p53 mutations in cancer. Nat Cell Biol 2013;15:2-8.

25. de Mello RA, de Vasconcelos A, Ribeiro RA, Pousa I, Afonso N, Pereira D, et al. Insight into p95HER2 in breast cancer: molecular mechanisms and targeted therapies. Recent Pat DNA Gene Seq 2012;6:56-63.

26. Thadani-Mulero M, Nanus DM, Giannakakou P. Androgen receptor on the move: boarding the microtubule expressway to the nucleus. Cancer Res
2012;72:4611-5.

27. Napetschnig J, Wu H. Molecular Basis of NF-kB Signaling. Annu Rev Biophys 2013; 42:443-68.

28. Miyahara S, Miyakoshi H, Yokogawa T, Chong KT, Taguchi J, Muto T, et al. Discovery of a novel class of potent human deoxyuridine triphosphatase inhibitors remarkably enhancing the antitumor activity of thymidylate synthase inhibitors. J Med Chem 2012;55:2970-80.

29. Zhang JJ, Inouye M. MazG, a nucleoside triphosphate pyrophosphohydrolase, interacts with era, an essential GTPase in Escherichia coli. J Bacteriol 2002;184: 5323-9.

30. Jackson RC. The regulation of thymidylate biosynthesis in Novikoff hepatoma cells and the effects of amethopterin, 5-fluorodeoxyuridine, and 3-deazauridine. J Biol Chem 1978;253:7440-6.

31. Pontarin G, Ferraro P, Hakansson P, Thelander L, Reichard P, Bianchi V. p53R2dependent ribonucleotide reduction provides Deoxyribonucleotides in quiescent human fibroblasts in the absence of induced DNA damage. J Biol Chem 2007; 282:16820-8.

32. Valinluck V, Liu P, Kang JI, Burdzy A, Sowers LC. 5-Halogenated pyrimidine lesions within a $\mathrm{CpG}$ sequence context mimic 5-methylcytosine by enhancing the binding of the methyl-CpG-binding domain of methyl-CpG-binding protein 2 (MeCP2). Nucleic Acids Res 2005;33:305764. 